\title{
STULTIFERA NAVIS: ACERCA DE LA ÚNICA ILUSTRACIÓN DEL PRIMER ESCRITO CHECO SOBRE AMÉRICA
}

A pesar de que la primera edición de El Elogio de la Locura (Mórias enkómioes, Stulticiae laus), de Erasmo de Rotterdam, escrita en 1509, fue publicada en 1511 y su traducción al checo ya en el año siguiente ', el término literario del elogio a la locura existía mucho antes. Su predecesor fue El Barco de los locos (Narrenshiff) del humanista de Estrasburgo Sebastián Brant, del año 1494, que se tradujo cinco años más tarde al latín; existe, también, su versión española ${ }^{2}$. Fue muy poco después del descubrimiento de América. Sin embargo, la idea del viaje alegórico de un barco fue conocida en la literatura ya desde el siglo XIv $^{3}$.

Un decenio más tarde, hacia el año 1506, fue publicada en la ciudad de Pilsen (Plzen), Reino de Bohemia, hoy República Checa, la primera información escrita en checo sobre el descubrimiento de América, de Mikulás Bakalár, bajo el nombre de Spis o nowych zemiech A o nowem swietie O niemzto gisme prwe zadne znamosti nemieli Ani kdy co slychali... ${ }^{4}$. (Escrito sobre nuevas tierras y sobre el mundo nuevo del cual no tuvimos antes ningunos conocimientos ni siquiera oímos de él...). En principio, se trata de la traducción adaptada del Mundus Novus de Amerigo Vespuccio ${ }^{5}$. Dado que se publicó en un país alejado, sin mar, hay que considerarlo como una reacción muy al día, que testimonia un interés por los viajes de descubrimiento a nivel del lector sólo en lengua checa, o sea, de hombre de a pie, pues el intelectual podía informarse en ediciones latinas, alemanas o italianas.

1 Edición checa Erasmus Rotterdamský, Chvála bláznivosti. List Martinu Dorpiovi (El Elogio de la Locura. Carta a Martín Dorpio). Mladá fronta, Praha 1986, p.7. Michal a Martin Svatos, Erasmus Rotterdamský, Zivá tvár Erasma Rotterdamského (Erasmus de Rotterdam. La Cara viva de Erasmus de Rotterdam), Vysehrad, Praha 1985, advirtieron un juego de palabras en el título del libro dedicado al humanista católico inglés ejecutado por el rey, Thomas More: el título Encomion Moriae id est Stultitiae laus pues contiene una referencia al nombre del amigo del escritor: Morus-Moriá (en griego locura).

${ }^{2}$ Michel Foucault, Dejiny sílenství. Hledáni historických korenú pojmu dusevní choroby (La historia de la locura. La búsqueda de las raíces históricas del concepto de la enfermedad mental, traducción al checo del original francés L'histoire de la folie a láge classique). Nakladatelství Lidové noviny, Praha, 1993, p. 13. Carlos Fuentes. «Prostor a cas Nového sveta» (El espacio y el tiempo del Nuevo Mundo, en checo), in: Svetová literatura XXXVII, 1992, nº 6, p. 122 advierte que la traducción al castellano de este escrito lo tenía ya en 1515 en su biblioteca el hijo del Descubridor, Hernando Colón ... Retraduciendo, intentaré reconstruir sus palabras que resumen de manera poética todo el problema: Tras Pinta, Niña y Santa María surcó la Nave de los locos, navis stultorum del famoso grabado de Brant. En el mástil vigilaba Machiavelli, Thomas More sería, en nuestra nave de los locos, timonero, y cartógrafo sería el medio jorobado y cauto Erasmo de Rotterdam. Sus consignas, sus banderas en el barco fueron -como sigue: este es, esto debe ser, esto puede ser ... Muchas veces resulta difícil -si no imposible- llegar a un buen puerto, pues la nave de los locos tropieza con los encallados del individualismo estoico que España heredó de Roma y lo trasladó a América ...

Véase también Isabel Mateo, «La temática de la Nave de los Locos en una edición española del S. XV». Traza y Baza 1973, n. 3 , pp. 45-51. A la autora le debo algunas observaciones acerca del tema que me transmite con su habitual amabilidad.

${ }^{3}$ James Hall, Slovník námetú a symbolu ve výtvarném umení (Diccionario de motivos y símbolos en las artes plásticas). Mladá fronta, Praha, 1991, p. 251.

${ }^{4}$ Spis o nových zemích a o novém svete. (Escrito sobre nuevas tierras y sobre el mundo nuevo del cual no tuvimos antes ningunos conocimientos ni siquiera oímos de él...). Facsímil y comentario del impreso de Pilsen de Mikulas Bakalar, del año 1506. Editado por Pravoslav Kneidl. Památník národního písemnictví, Praha 1981 (en español).

5 Spis ... op o cit. en la nota 4, p. 15. Véase también Jiri Hrubes, Nicolás Bakálar, impresor de Pilsen y primer informador sobre el Nuevo Mundo. Ibero-Americana pragensia, V, Praha 1970, pp. 209-215. Idem, Nicolás Bakalár, el primer portanuevas checo sobre los viajes de Descubrimiento. Ibero-Americana pragensia IX, 1975, pp.167-179. Recientemente menciona la importancia y la historia de este libro Ivan Sviták, First Bohemians in America. Prague 1992, pp. 4-5, reproducción en la contraportada. El grabado checo en cuestión está reproducido en varias publicaciones latinoamericanas, por ejemplo, Germán Arciniegas, América en Europa. Plaza y Janés, Bogotá 1982, p. 36, o Rafael Pineda, El Nuevo Mundo a los ojos del Nuevo Mundo. Caracas 1992. Como pura ilustración sin contexto alguno aparece en el trabajo de Lucila Anzola, Lecturas del Arte Nacional. Patrimonio fortificado durante el periodo colonial en Venezuela. Galería de Arte Nacional, Caracas, S.D., s.p. (p.3). Otra versión del grabado en madera pasó a ser emblema de la conferencia Spain and Portugal of the navigators. The Iberian Península, Europa and New Horizons, Washington, D.C., September, 25-29, 1990. Georgetown University Washington. Véase también la exposición eb la galería Hollar, Výtvarná práce (Praha), 1965, n. ${ }^{\circ}$ 16, p.2.

$A E A, \mathrm{LXXV}, 2002,297$, pp. 51 a 88 
En la portada de la edición checa encontramos un dibujo que representa un barco navegando por los mares con la vela tendida. Al bordo están de pie cuatro hombres (atrás apenas se distinguen otros), el quinto está en la popa: todos llevan amplios sombreros de peregrinos. El que manda parece ser el que está parado en medio de sus compañeros, en primer plano, con un bastón de peregrino en su mano derecha mientras que el brazo izquierdo lo está levantando con un gesto de predicar.

El grabado en madera anónimo fue creado sin duda como adopción libre del motivo del libro de Brant, ilustrado por Durero. Lo común es la composición del barco pero los locos de Brant son reemplazados por peregrinos ${ }^{6}$. Este dibujo pasó a ser emblema del anuario IberoAmericana Pragensia fundado por el profesor Josef Polisenský y publicado a partir del año 1967 por el Centro de Estudios Iberoamericanos de la Universidad Carolina en Praga ${ }^{7}$.

Pero, ¿qué fue lo que contribuyó a tal divulgación de esta ilustración-modelo? Su tradición se remonta al más allá de la época medieval. «En el paisaje quimérico del Renacimiento aparece un tema nuevo y rápidamente adquiere un puesto de preferencia: El Barco de los Locos, un raro navio ebrio surcando las aguas tranquilas de la región del Rin y de los canales flamencos» ${ }^{8}$.

En la misma época aparecen y se difunden composiciones como las de Symphorien Champier El barco de los Príncipes y de las Batallas Aristocráticas (1502), El barco de las Damas Honradas (1503), El barco de la Salud y, al lado de ellas, Blauwe Schute de Jacob van Oestvoren, del año 1513, así como la obra de Josef Bade Stultiferae naviculae scaphae fatuarum mulierum (1498). Lo que más coincide con el espíritu de la Nave de los Locos parece ser el libro que el inglés Alexandr Barclay publicó en 1509 «The ship of Folys», en el que expresó su repudio a profundizar los conocimientos geográficos y los descubrimientos ${ }^{9}$. También los cuadros de Jerónimo Bosco pertenecen a esta — según Foucault- «corriente de sueño» ${ }^{10}$.

Lo que pasa es que los barcos de los locos existían realmente en aquel entonces y los conocían todas las ciudades con el puerto marítimo y fluvial. Los marineros tenían como tarea liberar la ciudad de los locos llevándolos a otro sitio. De esta forma, los barcos de los locos

\footnotetext{
${ }^{6}$ Sebastián Brant, Albrecht Durer, Lod'Bláznu (La nave de los locos). Klub Prátel poezie, Ceskoslovenský spisovatel, Praha, 1972, ilustración en las guardas con el letrero/leyenda «Das Narren Schyff». Esta publicación presenta solamente una selección limitada de ilustraciones y textos (un cuarto). El presentador y traductor, Josef Brukner menciona que «todos los ejemplos de diferentes tipos de locos ... los encuadró Brant ... en una grata alegoría del Barco, navegando con su tripulación variada por el mar de la insensatez, necedad, brutalidad y arbitrariedad generales humanas. Y si su barco, a pesar de numerosos rompientes inesperados, pudo al final llegar hasta nuestras costas realmente tan lejanas, no lo logró gracias a su construcción, en cuyas armaduras carcomió palpablemente el tiempo, sino solamente gracias a su tripulación invencible y hasta inmortal, en medio de la cual se yergue con un mástil el propio Brant, este Noe de la época medieval, este Colón...» Un detalle de este grabado en madera lo utilizan como ilustración Michal a Martin Svatos, op. cit. En la nota 1, p. 236. Según María Antonieta Flores, «Discurso de la locura o discurso poético», Bajo Palabra, Caracas, III, 18-9-94, n. ${ }^{\circ} 115$, p.1, el loco representa el testimonio de la separación con la norma y con la concepción de la normalidad, imaginándonos bajo esta expresión cualquier cosa... En la locura se manifiesta de una forma más evidente la debilidad del «yo» frente al otro. La autora recuerda que la fascinación por la locura data por lo menos desde Platón quien expresó que los bienes más grandes proceden de la locura, otorgada sin duda alguna por un don divino. Platón recuerda en Faidros que el tercer estado, la locura, proviene de las musas.

${ }^{7}$ Hoy día dirigido por el Prof. Dr. Josef Opatrný

${ }^{8}$ Foucault, op. cit., en la nota 2, p. 12.

${ }^{9}$ Oldrich Kaspar, Nový svet v ceské a evropské literature 16-19 století (Nuevo mundo en la literatura checa europea). Universidad Carolina, Praha, 1980, p.48: «El interés por largos y arriesgados viajes crecía en Inglaterra poco a poco. E incluso fue objeto de crítica». Baraclay, sin embargo, valora «los descubrimientos desde el punto de vista de los conocimientos del reconocimiento del mundo y del anhelo superfluo y vanidoso por la gloria», y - agrega, en tono marxistaque «no los veía en el contexto económico» (véase nota 151 del citado libro de Kaspar, en el que hace referencia a Bohumil Bad'ura, «Nekteré aspekty spanelského a britského kolonialismu americe v 16. a v. 17. Století» (Algunos aspectos del colonialismo británico en América), in : Sborník k problematice dejin imperialismu (Miscelánea sobre los problemas de la historia del imperialismo). Praha 1973, s 9.

${ }^{10}$ Foucault, op. cit. en la nota 2, p. 13.
}

$A E A, \mathrm{LXXV}, 2002,297$, pp. 51 a 88 
atracando en las orillas pasaron a ser una imagen relativamente frecuente para las ciudades europeas. En algunos sitios, sobre todo en los de peregrinación, los locos se concentraban más que en otros. Puede que estos barcos que excitaban la fantasía del Renacimiento temprano, fueran incluso una especie de navíos de peregrinaje ${ }^{11}$. Los empeños por curar y por expulsar se unían en uno, en «el espacio sagrado del milagro» ${ }^{12}$. La ida de los locos se alineaba entre los exilios de carácter ritual. «El demente en su navío loco navega hacia otro mundo y de otro mundo también viene navegando» ${ }^{13}$.

Esta concepción la podemos relacionar también con el descubrimiento del Nuevo Mundo. Desde el mismo principio Colón fue considerado un soñador, por no decir un loco, ya que se decidió «por un acto que todas las edades consideraron como pura locura» ${ }^{14}$. «El loco ... es un Peregrino por excelencia. Y nadie conoce la tierra donde va a arribar» ${ }^{15}$.

Foucault responde a la pregunta de por qué esta repentina formulación del tema de los lo$\cos$ y de la locura en la literatura y pintura del siglo $\mathrm{xv}$, dando a saber su opinión de que se trata de un símbolo de una gran inseguridad que, de pronto, a fines de la época medieval surge en el horizonte de la cultura europea ${ }^{16}$. Es que, precisamente en aquel momento los europeos sobrepasaban la costa del mar en Europa como una frontera del miedo y, al mismo tiempo, de su esperanza ${ }^{17}$.

Si consideramos el arte como una dimensión espiritual de la sociedad, como una reflexión de la época de la vida de la sociedad y de su crisis moral, pues incluso la pintura «El barco de los locos del Bosco» (Louvre), en la que nos encontramos ante una compañía borracha, es un símbolo parecido del mundo en el cual la gente navega por el mar agitado y no se preocupa ni por el destino de su viaje ni por la llegada segura ${ }^{18}$. El sentido original de la simbología del barco surge de las ideas de los Padres y los apologetas que comparaban la Iglesia con el barco en el que los de buena fe encuentran la seguridad y que los conduce hacia la salvación ${ }^{19}$.

Sin embargo, el tema del barco es más complicado y a la vez más concreto. Si nos atenemos a la opinión del venezolano Rafael Pineda ${ }^{20}$, los niveles legendario y real se infiltran de

\footnotetext{
${ }^{11}$ Foucault, op. cit. en la nota 2, p. 14.

${ }^{12}$ Foucault, op. cit. en la nota 2, p. 14.

${ }^{13}$ Foucault, op. cit. en la nota 2, p. 15.

${ }^{14}$ Por otros véase Albert Vyskocil, Krystof Kolumbus. Zivot a význam objetivele globu (Cristóbal. Colón. Vida e importancia del Descubridor del Globo). SLK, Praha, 1945, p.14.

${ }^{15}$ Foucault, op. cit. en la nota 2, p. 17

${ }^{16}$ Foucault, op. cit. en la nota 2, ibid.

${ }^{17}$ Michel Mollat du Jourdin, Evropa a more (Europa y el mar). Bratislava, 1994, p. 13. Poco después, en 1536 -recuerda Mollat- el mapa del mundo, proyectado en una forma desacostumbrada, la de corazón, y la Europa con sus dimensiones modestas, está representada en medio del mapamundi en forma de la cabeza de payaso, con los atributos de la locura y varias lecturas de la consigna de la éppoca Vanitas vanitatum et omnia vanitas; una ilustración similar con el letrero Nosce te ipsum, fue realizada por Oronce Finé (p.120). «Recordemos que junto con una idea de carácter material y visual que difícilmente podamos esperar que madure en un país sin mar, se unía asimismo la imaginación humana que partía a veces de hechos y vivencias reales, otra vez de ideas preconcebidas y mitos. Su relación al mundo del mar tenía el valor de mensaje y de desafío. Resistiendo al tiempo y al espacio, el mar confronta su intemporalidad e infinito con la existencia pasajera de las generaciones humanas unidas a un solo pedazo de la tierra, como si el mar provocara por la inconstancia/ irregularidad de sus gustos al ser la tierra firme/seca, como si con la fuerza de su silencio resistiera al pulular humano e impusiera la humildad por su poder absoluto». (s. 234). Y Mollat sigue: «La Edad Media cristiana entendía el mar como un espacio que el Diablo quiere arrancarle a Dios. La convivencia del miedo y de la esperanza fue permanente. El mar se escapa a la ley humana» (p. 245). Pues, ya Erasmo opinaba que «no hay mayor locura que la de entregarse al mar» (p. 247).

18 J. Pesková, L. Schuckova, Já, clovek ... (Yo, el hombre), PN, Praha 1991, p. 193. Mia Cinotti, Arnaud Brejon de Lavergneé, Louvre, Paríz (Louvre, París). Bratislava 1989, p. 89, nº 128 lo interpretan como la «burla de la corrupción de la sociedad y del clero. Los motivos simbólicos están representados de una forma extraordinariamente real y reales son también los agitados gestos grotescos de los personajes repugnantes apretados en la barca».

${ }^{19} \mathrm{~J}$. Hall, op. cit. en la nota 3, p. 250.

${ }^{20}$ Rafael Pineda, La Utopía y cinco mitos americanos: El Dorado, las siete ciudades de Cibola, y el país de la CucañaJauja, Caracas 1989 (le agradezco el haberme proporcionado la copia).
} 
tal manera que no podemos distinguir uno del otro. Según James Hall ${ }^{21}$ en la poesía alegórica de Brant se narra sobre el barco lleno de locos que parten hacia «el paraíso de los locos» - Narragonia - De tal manera, la poesía de Brant sirve como medio para un comentario satírico de la locura de la época en un amplio sentido de la palabra: abarca la vida disipada, vicios de todo tipo, ebriedad, obscenidad, venalidad de los abogados, corrupción, etc.

En nuestro caso se añade un nivel de significado más: el timonel de la popa en el grabado de madera de Pilsen tiene en su sombrero una concha, el signo de peregrinos relacionado con el culto de Santiago y con las peregrinaciones, sobre todo a Santiago de Compostela ${ }^{22}$. Podemos entonces, considerar este barco representado en el primer escrito checo sobre el descubrimiento del Nuevo Mundo, inspirado en la ilustración que acompaña a la Nave de los Locos de Brant - conforme a las ideas arriba mencionadas y en el contexto de la edición checa- también como un barco de peregrinos que se dirige hacia la nueva tierra prometida, América, cuyos rasgos paradisíacos subrayó el descubridor mismo adhiriéndose al espíritu de la idealización utópica de la época, y por lo tanto, muy distante a la realidad ${ }^{23}$.

PAvel StepÁNeK

\section{CALIGRAFÍA Y GRABADO EN TORNO A D. PEDRO PAREDES}

Jonathan Richardson (1719) sugirió que el modo más apropiado de juzgar una obra de arte era situarla en el medio propio de la escuela local o en el período histórico al cual pertenecía. Pero igual de interesante es ver, en determinados hechos artísticos, la fuente de inspiración de tendencias actuales o la posible raíz de nuevas disciplinas.

La escritura es uno de los grandes inventos de la humanidad, es un lenguaje comunicativo que ha hecho posible la historia, siendo su inventor mítico el dios egipcio Tot. Es también algo cuyo dominio la mayoría de la gente da hoy por supuesto. Ya adultos, apenas si nos paramos a pensar en el método mental y físico gracias al cual nuestros pensamientos se codifican y transforman en símbolos sobre un soporte, sea una hoja de papel, una pantalla, o en bytes de información en un disquete de ordenador.

Mucho menos preocupa la técnica gráfica que se utiliza en estos procesos, o el ritmo corporal cuando se escribe.

Si se contempla la historia de la escritura es posible observar la riqueza que aúna este lenguaje, de paleta policroma, debido a la vasta influencia que ha tenido y que abarca muchas culturas e idiomas, prácticamente todos los períodos del desarrollo humano. Se sirve de ideas e información tomadas de una serie de disciplinas como Antropología, Arqueología, Historia del Arte, Economía, Lingüística, Técnicas de reproducción, Dibujo, Matemáticas, Historia política y social, Psicología y Teología, Literatura y manuscritos medievales, y sin ánimo de hacer interminable esta lista, alude a la caligrafía, a la tipografía y a la imprenta.

\footnotetext{
${ }^{21}$ J. Hall, op. cit. en la nota 3, p. 251.

${ }^{22}$ Viz Pavel Stepánek, Za sv.. Jakubem s «hyspánským strychem» pres Plzen. (En pos de Santiago con el «escudo hispánico» atravesando Pilsen). Ponencia presentada en la conferencia científica con ocasión de la exposición El gótico de la Bohemia Occidental en la Galería Nacional de Praga. En imprenta.

${ }^{23}$ Kolumbúv lodní deník (El cuaderno de bitácora de Colón. Con prefacio de Josef Susta. Novina, Praha, 1942, pp. 206214. Daniela Hodrová, Román zasvecení (La novela de consagración). Praha 1993, p. 190 recuerda que el espacio de iniciación de la Edad Media, el espacio interior en el modelo horizontal de la consagración, se expresa, entre otro, también con los puntos cardinales. En el Oeste se encuentran las islas y el imperio de los muertos, mientras que en el Este, el reino de Dios.
}

$A E A, \mathrm{LXXV}, 2002,297$, pp. 51 a 88 